\title{
Progress in the management of solid tumours
}

Series editor: Mr Colin D Fohnson, Department of Surgery, Southampton General Hospital, Southampton SO9 4WX, UK

\section{Soft tissue tumours}

\author{
Andrew J Hayes, J Meirion Thomas
}

\section{Summary}

Any soft tissue swelling beneath the deep fascia should be considered a sarcoma until proven otherwise. As the most important factor in the primary treatment of these cancers is the adequacy of the primary surgical resection, it is vital to diagnose these malignant tumours pre-operatively.

The modern treatment of soft tissue sarcomas may involve all modalities, but the most important aspect of treatment of a primary localised sarcoma is wide excisional surgery preserving limb function. Radiotherapy is a vital adjunct in high-grade tumours, or in tumours whose resectability is limited either by size or anatomical proximity to vital structures. Apart from a few chemosensitive sarcomas, the role of chemotherapy is limited to treatment of metastatic disease where documented response rates are no greater than $30 \%$. As $50 \%$ of patients with high-grade sarcomas will die from metastatic disease, improvements in survival rates will only come from improvements in response to systemic therapy. No controlled trials have shown any survival benefit for adjuvant chemotherapy, although a recent meta-analysis of published data has shown a trend to increased survival at two years. Multicentre randomised trials are ongoing.

The prognosis of these lesions is highly variable, but is intimately related to the anatomical site (ie, resectability), and also the grade and size of the tumour.

Keywords: soft tissue tumour, sarcoma

The Sarcoma Unit, The Royal Marsden Hospital, Fulham Road, London SW3

3JJ, UK

AJ Hayes

JM Thomas

Correspondence to Professor Thomas

Accepted 30 October 1996
Soft tissue sarcomas are rare cancers, accounting for $1 \%$ of all malignant tumours. ${ }^{1}$ Benign tumours of soft tissue can mimic primary soft tissue malignancies. Over a five-year period 856 patients were referred to our unit with a presumed diagnosis of a primary soft tissue malignancy, of which 207 were eventually proven to be benign tumours. The most common diagnosis was fibromatosis $(30 \%)$, but other lesions also encountered included intramuscular lipomas (19\%), arteriovenous malformations (8\%), nodular fasciitis (7\%), myxoma $(6 \%)$ and neurofibromas $(6 \%)$. Similarly, other malignancies can present as masses within the soft tissues, particularly lymphoma, bony malignancies and metastatic carcinoma.

Although the incidence of soft tissue sarcoma is increased in some inherited conditions, the majority of cases are sporadic with no clear aetiology. Documented familial associations are von Recklinghausen's disease, ${ }^{2}$ familial retinoblastoma and the Li-Fraumeni syndrome, where there is inherited mutation in the tumour suppressor gene p53. ${ }^{3}$ Therapeutic irradiation and chronic lymphoedema are non-inherited conditions which are associated with an increased incidence of soft tissue sarcoma, ${ }^{4,5}$ usually after a lengthy interval. No environmental carcinogens have been convincingly associated with the development of soft tissue sarcomas, although the chemical dioxin has been implicated on tenuous historical evidence. ${ }^{6}$

\section{Pathology}

\section{HISTOPATHOLOGY}

Soft tissue tumours are proliferations of mesenchymal as opposed to epithelial tissue and thus there is a great diversity of cellular appearance, depending upon the line of differentiation of the mesenchymal element of origin (box 1). There are, however, some general patterns of surgical pathological behaviour, irrespective of cell type:

- the growth of the primary tumour tends to be limited to within a fascial compartment and extend along it, and the fascial or periosteal boundaries are rarely breached,

- they exhibit a pseudo-capsule which gives the macroscopic impression of encapsulation, although microscopically tumour cells infiltrate and extend beyond this layer,

- the site of metastatic spread is usually to lung and rarely to lymph nodes.

Pathological classification centres around these major cellular elements (eg, lipomatous tumours, fibrohistiocytic tumours, smooth muscle tumours). ${ }^{7}$ As well as the morphology of the cells, immunohistochemistry has enhanced the pathologist's ability to categorise the tumour by identifying cellular elements that are specific to particular lines of differentiation, eg, the intermediate filament desmin in tumours of muscular origin and the nervous tissue antigen S-100 in neurogenic tumours. ${ }^{8}$ Therefore, purely morphological classifications such as round cell or spindle cell sarcomas are outdated. Cytogenetic analysis identifying particular chromosomal translocations are of diagnostic value in some soft tissue sarcomas such as primitive neuroectodermal tumours, extra skeletal Ewing's tumour, and synovial sarcoma. ${ }^{9}$

\section{GRADING AND STAGING}

The grade of a tumour is a more important determinant of malignant potential than the cell type. A number of grading systems have been developed that score tumours on features such as cellularity, mitotic rate, differentiation and necrosis to classify it as low, intermediate, or high grade. ${ }^{10}$ The grade of tumour, its size at presentation and the presence of regional or distant metastasis are the usual 


\begin{tabular}{|l|}
\hline $\begin{array}{l}\text { Frequency of the major } \\
\text { histological types of } \\
\text { sarcoma (from }\end{array}$ \\
\hline - malignant fibrous histiocytoma: \\
\\
$21 \%$ \\
- liposarcoma: $17 \%$ \\
- leiomyosarcoma: $19 \%$ \\
- synovial sarcoma: $11 \%$ \\
- malignant nerve sheath tumour: $6 \%$ \\
rhabdomyosarcoma: $5 \%$
\end{tabular}

Box 1

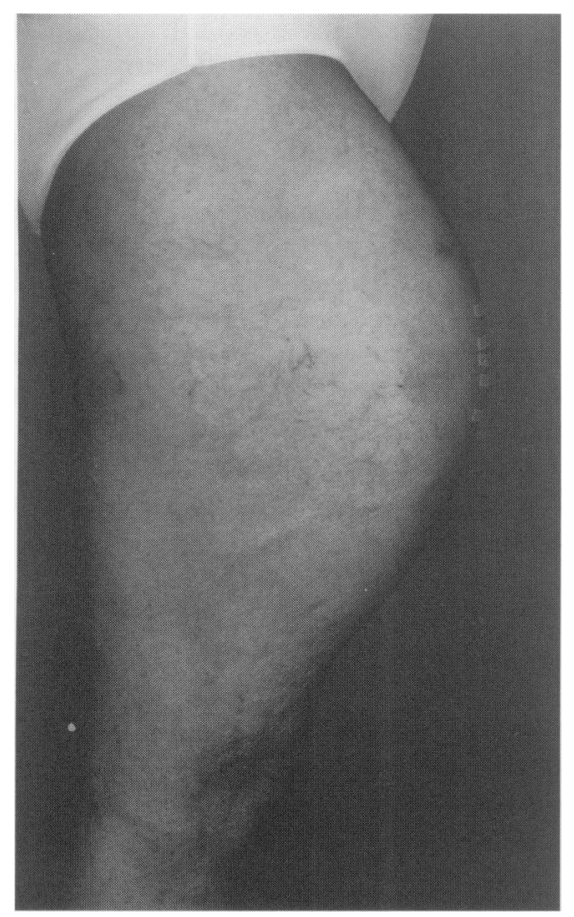

Figure 1 The clinical appearance of a massive soft tissue sarcoma in the quadriceps compartment

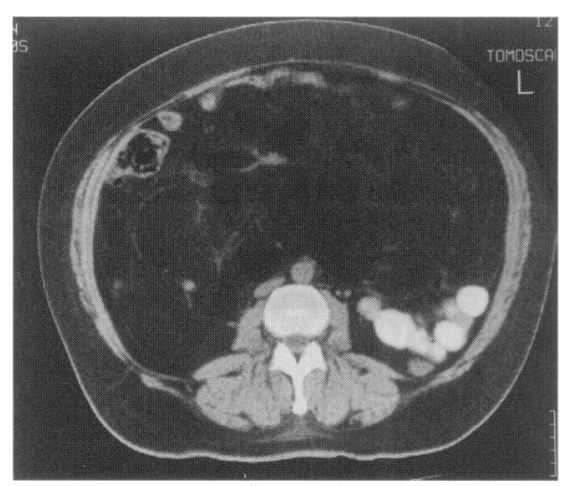

Figure 2 A huge retroperitoneal low to intermediate grade liposarcoma. This patient was asymptomatic other than for noting progressive abdominal distension factors identified in staging sarcomas. The authors believe that any staging system must take account of site, as anatomical proximity to vital structures may prevent wide excisional surgery with an adverse effect on prognosis. Enneking has proposed such a staging system. ${ }^{11}$

\section{Diagnosis}

PRESENTATION AND CLINICAL FEATURES

The majority of soft tissue sarcomas are on limbs or limb girdles ${ }^{12}$ and the patient presents having noticed a painless enlarging swelling, usually within a large muscle group (figure 1). The most common site of sarcoma is in the quadriceps compartment. Retroperitoneal sarcomas account for approximately $20 \%$ of all soft tissue sarcomas ${ }^{13}$ and usually present late, often when the tumour has reached a considerable size (figure 2). Pain is very uncommon although occasionally referred pain or dysthasthesia in the leg is seen because of compression of the femoral or sciatic nerves within the pelvis. ${ }^{14}$ Similarly, symptoms of bowel obstruction are uncommon and often the patient will present only when the tumour has reached sufficient size to cause noticeable abdominal distension.

Careful clinical examination can accurately identify the size and local relations of the soft tissue sarcomas on the limbs. The tumours tend to be limited to one fascial compartment (figure 3) and only rarely involve neurovascular structures. Consequently, signs or symptoms of neurovascular insufficiency or clinical evidence of skin involvement usually indicate advanced or aggressive disease. Deep fixation to bone is uncommon in all but the most advanced tumours, but tight confinement of the tumour within a deep fascial compartment can give the impression, on clinical examination, of deep bony attachment.

It is rare for soft tissue sarcomas to present with clinical evidence of distant disease. The commonest site for metastatic spread is the lungs and this is usually asymptomatic at presentation. In our unit approximately $12 \%$ had metastatic disease in the chest at the time of presentation, ${ }^{13}$ the majority of which were only evident radiologically. Malignant lymphadenopathy can be seen at presentation, but this is rare and usually indicates a poor prognosis.

\section{INVESTIGATION OF SUSPECTED SOFT TISSUE SARCOMAS}

Because these cancers are rare, they should be referred to specialist units, where much radiological, histopathological and clinical experience exists. Ideally, investigation and treatment should be performed in the same centre to avoid delay in diagnosis and also to prevent inappropriate or unnecessary procedures.

The two central aspects of investigation (box 2) are obtaining an accurate pre-treatment tissue diagnosis and imaging of the primary soft tissue tumour.

\section{Tissue diagnosis}

This is obtained most readily by Trucut ${ }^{\mathrm{k}}$ biopsy. This can be performed easily in an out-patient department under local anaesthesia. The biopsy site should be in an area of skin that will be excised in the definitive operation. We have

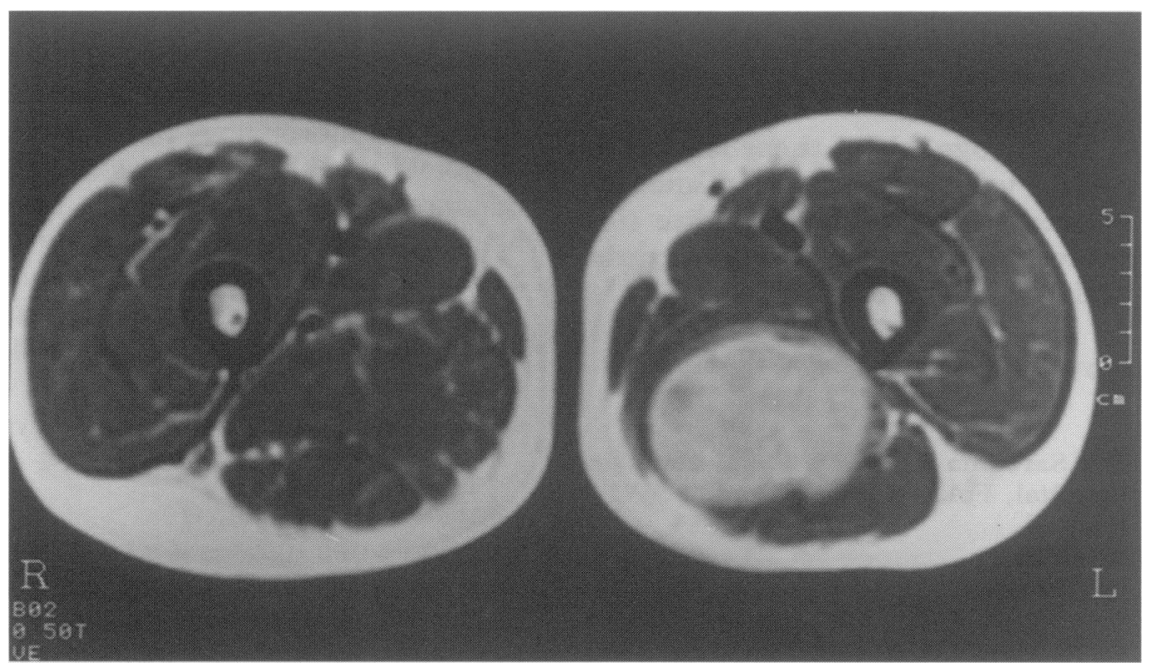

Figure 3 MRI scan showing a soft tissue sarcoma arising in the hamstring compartment 


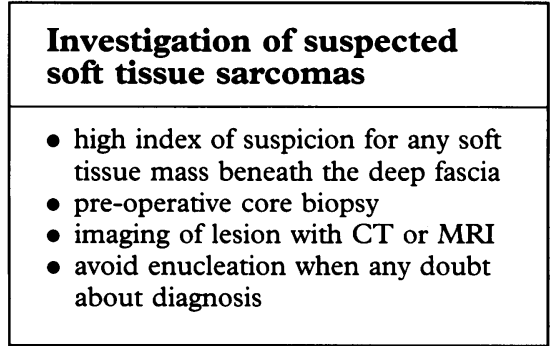

Box 2

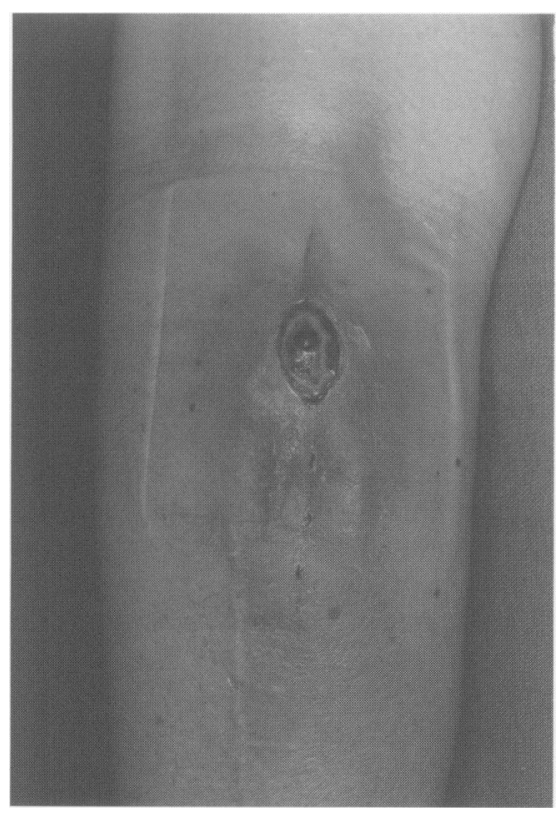

Figure 4 The hazards of open biopsy. This patient developed spreading cellulitis with tumour ulcerating through the skin after an open biopsy

\begin{tabular}{l} 
Surgical resection of soft \\
tissue sarcoma \\
\hline - intracapsular, where the tumour is \\
enucleated or the pseudo-capsule is \\
breached \\
- marginal, in which the margin of \\
excision is the level of the pseudo- \\
capsule \\
wide, where the resection of the \\
tumour includes en bloc excision of \\
normal muscle tissue surrounding \\
the tumour but leaving some \\
functional muscle within the \\
compartment \\
radical, where an entire anatomical \\
compartment within its fascial \\
envelope is resected. Radical \\
amputational surgery for trans- \\
compartment tumours involves a \\
disarticulation at the joint proximal \\
to the level of the muscular \\
compartment containing the tumour
\end{tabular}

reported accuracy rates of $94 \%$ in diagnosing soft tissue sarcomas and $98 \%$ in identifying the tumour as malignant. The correct subtype of sarcoma was diagnosed in $85 \%$ of cases. ${ }^{15}$ Fine-needle aspiration has been shown to be less sensitive than Trucut biopsy, with one study demonstrating a false-negative rate of $18.5 \% .^{16}$ Fine-needle aspiration may be useful in the diagnosis of local recurrence or regional lymphadenopathy when the small size of the abnormality preludes a Trucut biopsy.

Open biopsy is rarely necessary and should only be considered after a Trucut biopsy has failed to yield a conclusive diagnosis on two occasions. If an open biopsy is performed it should be performed by the surgeon responsible for the definitive operation. Complication rates of up to $17 \%$ have been reported in series of open biopsies and we would therefore not advise this as a primary diagnostic manoeuvre. ${ }^{19}$

Primary enucleation of a soft tissue sarcoma as a means of obtaining a histological diagnosis must be considered a grave error of management. These tumours possess a pseudo-capsule and therefore, although they may give the impression of being well encapsulated, malignant cells extend well beyond this artificial boundary. Consequently, after such a procedure a secondary excision of the tumour bed is necessary and it is often very difficult to identify the original site of the tumour once it has been surgically enucleated.

\section{Imaging}

Imaging of a soft tissue tumour is important as a complementary diagnostic investigation alongside biopsy and is vital in determining resectability, surgical planning and identifying metastatic pulmonary disease. The plain film is a simple investigation which may provide valuable information and should not be ignored despite the obvious superiority of computed tomography (CT) and magnetic resonance imaging (MRI) in imaging the primary tumour. Occasionally, periosteal reactions may be seen which could indicate direct bony involvement and bony metastatic disease can be identified as areas of bone lucency.

Definitive imaging of soft tissue tumours is by CT or MRI. Some comparative studies have demonstrated a superiority of MRI over CT in outlining the extent and relationships of soft tissue tumours, particularly to neurovascular structures. ${ }^{18}$ However, a contrast-enhanced CT scan remains an excellent investigation for identifying the extent of the tumour and its relationship to major vessels. Angiography is rarely indicated. Furthermore, CT scanning rather than MRI remains the investigation of choice for identifying the presence of pulmonary metastases as it has a much greater resolution than a simple chest X-ray and this can be performed at the same time as imaging the primary tumour. Neither CT nor MRI can reliably distinguish benign from malignant soft tissue tumours in all situations, further emphasising the need for a pre-operative tissue biopsy.

\section{Treatment}

ISOLATED SURGERY FOR EXTREMITY PRIMARY TUMOURS

Wide surgical excision is the mainstay of primary treatment for isolated primary disease affecting the limbs or limb girdles. Enneking ${ }^{11}$ has classified the surgical resection of soft tissue sarcomas into four categories (box 3).

Local recurrence rates are closely related to the adequacy of the primary surgery. ${ }^{19}$ Intracapsular resection will result in $100 \%$ of cases recurring locally, while marginal resections will result in local recurrence rates of $80-90 \%$ because the resection has been performed at the level of a pseudo-capsule with malignant cells lying distant to this artificial margin. Local recurrence rates for wide and radical surgery have been reported as approximately $40 \%$ and $20 \%$, respectively. ${ }^{20}$ Because of these very different local recurrence rates and also the very different level of disabilities resulting from radical versus more conservative surgery, there have been differing proposals for the surgery for a primary intracompartmental tumour. Enneking proposed a total compartmentectomy, ${ }^{11}$ whereas other authors have recommended that the tumour should be excised with a $2-3 \mathrm{~cm}$ margin of surrounding normal tissue. Following on from work by Karakousis, ${ }^{21}$ we have recently described a functional compartmental resection for tumours arising in the quadriceps or buttock compartments in which the tumour is excised radically, but preserving one uninvolved muscle within that compartment together with its innervation. Such an approach will in many cases produce subjectively normal strength in that muscle compartment after rehabilitation, whilst not compromising the radicality of the resection. ${ }^{22}$ When the tumour is clearly confined within one single muscle we simply excise that muscle from insertion to origin ensuring a plane of normal muscle tissue around it. ${ }^{13}$ 
ADJUVANT RADIOTHERAPY

The combination of wide excisional surgery and postoperative radiotherapy has resulted in local recurrence rates similar to those seen after radical surgery or amputations. ${ }^{23}$ Adjuvant radiotherapy is of particular importance when the tumour arises in a site where wide excision is precluded because of neighbouring vital structures. In our unit adjuvant radiotherapy is given to all patients with high-grade tumours and to any intermediate grade soft tissue sarcoma where wide excision is in question. Traditionally, radiotherapy is given in the early postoperative period as there is no convincing evidence of an advantage in pre-operative radiotherapy, and a detrimental effect on wound healing, particularly in the elderly, has been reported..$^{24}$

\section{RETROPERTONEAL SARCOMAS}

Retroperitoneal sarcomas have a poor prognosis with reported overall five-year survival rates are in the order of $20 \% .{ }^{25}$ Two reasons have been identified for the particularly poor prognosis in this subgroup. Firstly, the tumours arise in very close proximity to vital vessels or viscera which prevents complete surgical clearance in many cases. Numerous studies have identified complete excision of retroperitoneal sarcomas as being correlated with overall survival and yet this is often precluded by the site of disease. Secondly, the limited tolerance of surrounding structures to radiotherapy, especially small bowel, kidney and spinal cord, exclude this form of treatment as effective adjuvant therapy.

ADJUVANT CHEMOTHERAPY FOR ISOLATED PRIMARY SOFT TISSUE SARCOMAS Despite adequate primary treatment of soft tissue sarcomas that present without evidence of metastatic disease, $50 \%$ of patients with high grade tumours will die from metastatic disease. ${ }^{26}$ Consequently, there is great interest in the value of adjuvant chemotherapy and its ability to influence survival. To date there have been 15 published randomised trials comparing adjuvant chemotherapy with no chemotherapy. All of these trials were small and therefore lacked the statistical power to demonstrate any difference in survival unless this difference was really very striking. However, a recent meta-analysis performed by Tierney et al ${ }^{27}$ has demonstrated an improvement in survival at two years in favour of chemotherapy. The authors are cautious in interpreting this evidence as the individual patient data were not analysed consistently from trial to trial. Until such an analysis is complete or positive evidence from a larger randomised trial with greater power is published, one must conclude that there has been no proven benefit for treating isolated primary tumours with adjuvant chemotherapy.

\section{Treatment of metastatic soft tissue sarcoma}

\section{CHEMOTHERAPY}

As noted above, up to $12 \%$ of patients with soft tissue sarcomas will present with evidence of metastatic disease, usually after imaging of the chest. ${ }^{13}$ The response of soft tissue sarcomas to chemotherapy in all but a few subtypes (Ewing's sarcoma, primitive neuroectodermal tumour) is extremely disappointing. Single agent adriamycin remains the most effective agent with reported response rates ranging from $15-35 \% .{ }^{28}$ There has been some evidence of an increased response with increasing doses but there is little evidence suggesting that combination therapy is of any greater efficacy. One encouraging area is the combined use of adriamycin and ifosfamide together with bone marrow support with GM-CSF to prevent myelosuppression. Response rates of up to $45 \%$ have been reported with this combination. ${ }^{29}$ However, there is no convincing evidence that long-term survival is increased and therefore this treatment is essentially palliative.

Primary excisional surgery is often appropriate as first-line treatment for patients with an extremity tumour and asymptomatic pulmonary metastases, particularly as these high-grade tumours can go on to cause distressing local symptoms because of their size, and skin ulceration.

\section{METASTASECTOMY}

The value of metastasectomy in pulmonary metastatic disease is controversial. A number of series have demonstrated a five-year survival rate after pulmonary metastasectomy of between 20 and $40 \% .^{30}$ However, these series are highly selected and may well be quite unrepresentative of the entire spectrum of pulmonary metastatic disease. Gadd et al have reported their total experience of pulmonary metastatic disease in 716 patients who presented with primary extremity soft tissue sarcoma. ${ }^{31}$ Of these patients, 135 developed pulmonary metastasis and 55 of these had their metastasis completely resected; 15 of these 


\begin{tabular}{|l|}
\hline Management of soft tissue \\
sarcomas \\
\hline - histopathological diagnosis and \\
primary treatment are best \\
performed in specialist units with \\
wide experience of sarcomas \\
- wide excisional surgery and adjuvant \\
radiotherapy produces excellent \\
local control and good postoperative \\
function in extremity sarcomas \\
sarcomas in which wide excision is \\
limited by anatomical structures, eg, \\
in the retroperitoneum, have a \\
poorer prognosis \\
chemotherapy is currently only \\
indicated in metastatic disease, \\
though its role in the adjuvant \\
setting is under investigation in \\
clinical trials \\
\hline
\end{tabular}

Box 4 were alive at three years. This is $11 \%$ of the patients who developed pulmonary metastasis and $2 \%$ of the total patient population. Pulmonary metastasectomy has not been evaluated in a randomised study. Nevertheless, within published series, long-term survival rates of up to $20 \%$ for metastatic soft tissue sarcomas have been reported in a condition which is presumed to be almost invariably fatal without treatment. The prognostic factors which are thought to indicate a poor response to pulmonary metastasectomy are numerous metastases and a short disease-free interval from resection of the primary tumour.

\section{Prognosis}

Because the spectrum of tumours has such a variable clinical course from highly aggressive to relatively indolent, there have been many attempts to identify factors which relate to poor long-term survival. We are particularly interested in independent prognostic factors, rather than factors which appear to have prognostic significance, but are in fact simply a consequence of other, more important influences (eg, proximal limb tumours may appear to have a worse prognosis than distal limb tumours simply because they present at a later stage and greater size as they lie in bulky muscle groups, the site itself having no influence on survival). Independent factors relating to poor overall survival are high tumour grade, ${ }^{32}$ large tumour diameter, ${ }^{33}$ regional lymph node metastases $^{32}$ and failure to achieve adequate primary surgical clearance. ${ }^{34}$

\section{Future directions}

Limb sparing, wide excisional surgery and adjuvant radiotherapy have resulted in excellent local control rates and limb function whilst not compromising cure. Pre-operative diagnosis by core biopsy and treatment within specialist units will minimise inadequate primary surgical excisions. However, a large number of patients will go on to die from pulmonary metastatic disease regardless of effective local treatment. There are no effective chemotherapeutic agents for metastatic sarcomatous disease. Improvements in survival will stem from improvements in chemotherapy in the adjuvant and metastatic setting.

14 Ball ABS, Serpell JW, Fisher C, Thomas JM Primary soft tissue tumours of the pelvis causing referred pain in the leg. $\mathcal{F}$ Surg Oncol 1991; 47: $17-20$

1 Enzinger FM, Weiss SW. Soft tissue tumours, 3rd edn. St. Louis: Mosby. 1995; pp $1-7$.

2 Sorensen SA, Mulvihill J, Neilsen A. Long-term follow up of von Recklinghausen neurofibromatosis. N Engl f Med 1986; 314: 1010-5.

$3 \mathrm{Li} \mathrm{FP,} \mathrm{Fraumeni} \mathrm{JP.} \mathrm{Soft} \mathrm{tissue} \mathrm{sarcomas,} \mathrm{breast}$ cancer and other neoplasms : a familial syndrome? Ann Intern Med 1969; 71: 474-51.

4 Stewart FW, Treves N. Lymphangiosarcoma in postmastectomy lymphedema. Cancer 1948; 1: 68-81

5 Pitcher ME, Davidson ID, Fisher C, Thomas JM. Post irradiation sarcoma of soft tissue and bone. Eur f Surg Oncol 1994; 20: 53-6.

6 Suruda AJ, Ward EM, Fingerhut MA. Identification of soft tissue sarcoma deaths in cohorts tion of soft tissue sarcoma deaths in cohorts exposed to dioxin and to chlorina

7 Chung EB. Current classification of soft tissue tumors. In: Fletcher CDM, McKee PH, eds, Pathology of soft tissue tumours, Edinburgh Churchill Livingstone, 1990; pp 43-81.

8 Mukai $M$, Torikata $C$, Iri $H$. Immunohistochemistry of soft tissue tumours. In: Fletcher CDM, McKee PH, eds. Pathobiology of soft tissue tumours, Edinburg

9 Fletcher JA. Cytogenetics In: Verweij J, Pinedo HM, Suit HD, eds, Multi-disciplinary treatment of soft tissue sarcomas, Boston: Kluwer Academic soft tissue sarcomas, Boston:
Publishers, 1993; pp 23-35.

10 Coindre JM, Trojani M, Contesso G, et al. Reproducibility of a histopathological grading system for adult soft tissue sarcoma. Cancer 1986; 58: $306-9$

11 Enneking WF, Spanier SS, Malawar MM. The effect of the anatomic setting on the results of surgical procedures for soft part sarcoma of the thigh. Cancer 1981; 46: 1005-22.

12 Rantakokko V, Efkors TO. Sarcomas of the soft tissues in the extremities and limb girdles. Acta Chir Scand 1979; 145: $385-94$.

13 Pitcher ME, Thomas JM. Management of soft tissue sarcoma. Br f Surg 1994; 81: 1136-9.
15 Ball ABS, Fisher C, Pittman M, Watkins RM, Westbury G. Diagnosis of soft tissue tumours by Trucut ${ }^{r}$ biopsy. $B r \mathcal{F}$ Surg 1990; 77: 756-8.

16 Kissin MW, Fisher C, Webb AJ, Westbury G. Value of fine needle aspiration in the diagnosis of soft tissue tumours: a preliminary study on the excised specimen. Br f Surg 1987; 74: 479-80

17 Mankin HJ, Lange TA, Spanier SS. The hazards of biopsy in patients with malignant primary bone and soft tissue tumour. $\mathcal{f}$ Bone foint Surg ( $\mathrm{Am})$ 1982; 64A: $1121-7$.

18 Tehranzadeh J, Mnaymneh W, Ghavam C Morillo G, Murphy BJ. Comparison of CT and MR imaging in musculoskeletal neoplasms. Comp Assist Tomogr 1989; 13: 466-72.

19 Stotter AT, A'Hern RP, Fisher C, Mott AF Fallowfield ME, Westbury G.The influence of on metastasis and survival. Cancer 1990; 60: 1119-29.

20 Simon MA, Enneking WF. The management of soft tissue sarcomas of the extremities. $\mathcal{F}$ Bone foint Surg ( $\mathrm{Am})$ 1976; 58A: 317-27.

21 Karakousis CP. Modified anterior compartment resection. f Surg Oncol 1991; 46: 25-30.

22 Pitcher ME, Thomas JM. Functional compartmental resection for soft tissue tumours. Eur $\mathcal{F}$ mental resection for soft tiss
Surg Oncol 1994; 20: 441-5.

23 Lindberg RD, Martin RG, Rohmsdahl MM, Barkley HT. Conservative surgery and postoperative radiotherapy in 300 adults with sof tissue tumors. Cancer 1981; 47: 2391-7.

24 Ball ABS, Robinson M, Harmer C, Thomas JM Preoperative radiotherapy to facilitate surgery fo soft tissue sarcoma of the limbs. Eur $\mathcal{F}$ Surg Oncol 1997 ; in press.

25 Jenkins MP, Alvaranga JC, Thomas JM. The management of retroperitoneal soft tissue sarcomas. Eur $\mathcal{f}$ Cancer 1996; 32A: 622-6. local recurrence of extremity soft tissue sarcoma
26 Potter DA, Kinsella T, Glatstein E, et al. High Cancer 1986; 58: 190-205.

27 Tierney JF, Mosseri V, Stewart LA, Souhami RL, Parmar MKB. Adjuvant chemotherapy for soft tissue sarcoma: review and meta-analysis of the published results of randomised clinica trials. $B r$ F Cancer 1995; 72: 469-79.

28 Creagan ET, Hahn RG, Ahmann DL, et al. A clinical trial of adriamycin (NSC 123127) in advanced sarcoma. Oncology 1977; 34: 90-1.

29 Steward WP, Verweij J, Somers R, et al. Granulocyte macrophage colony-stimulating factor allows safe escalation of dose intensity in metastatic adult soft tissue sarcoma: a study of the EORTC Soft Tissue and Bone Sarcoma the EORTC Soft Tissue and Bone
Group, $\mathcal{f}$ Clin Oncol 1993; 11: 15-21. of prognostic factors in patients undergoing resection of pulmonary metastases from sof tissue sarcoma. $\mathcal{f}$ Cardiovasc Surg 1984; 87: 260-8.

31 Gadd MA, Casper SE, Woodruff JM, McCormack PM, Brennan MF. Development and treatment of pulmonary metastasis in adult patients with extremity soft tissue sarcoma. Ann Surg 1993; 218: 705-12.

32 Collin C, Godbold J, Hadju S, Brennan $M$. Localized extremity sarcoma: an analysis of factors affecting survival. $\mathcal{f}$ Clin Oncol 1987; 5: $601-12$.

33 Ruka W, Emrich D, Driscoll DL, Karakousis CP. Clinical factors and treatment parameters affecting prognosis in adult high-grade soft tissue sarcomas: a retrospective review of 267 cases. Eur ₹ Surg Oncol 1989; 15: 411-23.

34 Robinson M, Barr L, Fisher C, et al. Treatment of extremity sarcoma with surgery and radiotherapy. Radiother Oncol 1990; 18: 221 -3. grade soft tissue sarcomas of the extremities.

30 Puttnam JB, Roth JA, Wesley MN, et al. Analysis 